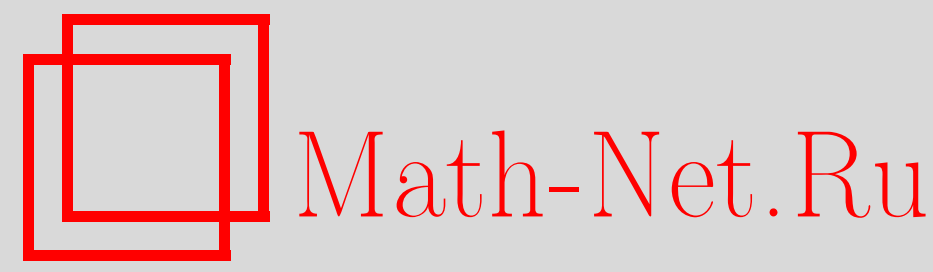

С. Трубецкой, Й. Шмелинг, Неоднородные диофантовы приближения и угловая возвращаемость для бильярдов в многоугольниках, Матем. сб., 2003, том 194, номер 2, 129-144

DOI: https://doi.org/10.4213/sm717

Использование Общероссийского математического портала Math-Net.Ru подразумевает, что вы прочитали и согласны с пользовательским соглашением

http://www . mathnet.ru/rus/agreement

Параметры загрузки:

IP: 54.196 .121 .252

26 апреля 2023 г., 09:12:03 


\title{
Неоднородные диофантовы приближения и угловая возвращаемость для бильярдов в многоугольниках
}

\begin{abstract}
Для заданного числа вращения вычисляется хаусдорфова размерность множества хорошо приближаемых чисел. Этот результат вместе с неоднороднњгм вариантом теоремы Ярника исползуется для доказательства свойств сильной возвращаемости бильярдного потока в некоторых многоугольниках.

Библиографиия: 16 названий.
\end{abstract}

\section{§1. Введение}

За последние десять лет в четырех независимых статьях было замечено, что бильярдная траектория произвольной точки, начинающаяся перпендикулярно стороне многоугольника и в какой-то последующий момент времени попадающая на некоторую сторону под прямым углом, проходит свой путь бесконечно часто в обоих направлениях между этими перпендикулярньми соударениями и потому является периодической. Наиболее ранняя из этих статей, принадлежашая Рюджгроку [1], вычислительная; она содержит гипотезу, что в каждом треугольнике есть перпендикулярные периодические траектории. В 1992 г. Бошерницан [2] и, независимо от него, Воробец, Гальперин и Степин [3] доказали, что бильярдная траектория, начинающаяся перпендикулярно любой стороне любого рационального многоугольника, является периодической для всех начальных точек на этой стороне, кроме конечного числа. Наконец, Сипра, Хансон и Колан [4] рассмотрели траектории, выходящие из точек одного из катетов иррационального прямоугольного треугольника перпендикулярно этому катету, и показали, что почти для всех таких точек (в смысле линейной меры Лебега на стороне треугольника) указанные траектории периодические. Использованньй ими метод доказательства фактически приводит к более сильному результату: в любом (обобщенном) параллелограмме или прямоугольном треугольнике и для любого направления $\theta$ множество $F_{\theta}$ тех точек, траектории которых, выходящие в направлении $\theta$, никогда не возвращаются параллельно самим себе, имеет нулевую меру Лебега [5]. Результат Сипры и др. о периодических траекториях следует из этого наблюдения. Проведенное ими компьютерное моделирование подсказьвает, что утверждение о "почти всюду", возможно, поддается улучшению. Это и является отправной точкой нашего исследования. В теореме 4.1 показывается, что в любом (обобщенном) параллелограмме и для любого направления $\theta$ нижняя bох-размерность множества точек, траектории которых, выходящие в направлении $\theta$, никогда не возвращаются параллельно самим себе, не превосходит $1 / 2$. В качестве следствия получаем утверждение, что 
bох-размерность множества точек катета прямоугольного треугольника, траектории которых, начинающиеся перпендикулярно катету, не являются периодическими, не превосходит $1 / 2$.

Затем мы переходим к вопросу о том, существуют ли направления $\theta$, для которых постоянная $1 / 2$ в теореме 4.1 может быть улучшена. Теорема 4.4 утверждает, что если $\mu>1$ есть порядок аппроксимации направления $\theta$ числом $\alpha$, то нижняя bох-размерность множества $F_{\theta}$ в действительности не превосходит $(\mu+1)^{-1}$. Имеются два приложения теоремы 4.4. Рассмотрим множество $\mathscr{C}_{s}$ направлений $\theta$, для которых множество точек, траектории которых никогда не возвращаются параллельно самим себе, имеет нижнюю bох-размерность не более $s \in[0,1 / 2]$. Теорема 4.6 утверждает, что хаус дорфова размерность множества $\mathscr{C}_{s}$ не меньше $s /(1-s)$, причем $\mathscr{C}_{s}-$ остаточное (т.е. плотное $G_{\delta^{-}}$) множество bох-размерности 1 . Зафиксируем направление $\theta_{0}$ и рассмотрим множество $\mathscr{D}_{s}\left(\theta_{0}\right)$ таких углов $\alpha$, что для любого обобщенного параллелограмма с углом $\alpha$ размерность множества точек, траектории которых, выходящие в направлении $\theta_{0}$, никог да не возвращаются параллельно самим себе, не превосходит $s \in[0,1 / 2]$. Рассмотрим также множество $\mathscr{E}_{s}$ прямоугольных треугольников, для которых размерность множества непериодических траекторий, перпендикулярных заданному катету, не превосходит $s \in[0,1 / 2]$. Теорема 4.7 устанавливает, что хаусдорфовы размерности множеств $\mathscr{D}_{s}$ и $\mathscr{E}_{S}$ не меньше $2 s$, причем эти множества - остаточные и имеют ящичную размерность 1 .

Доказательства теорем 4.6 и 4.7 происходят из чисто теоретико-числовых рассуждений. Аппроксимация угла $t$ кратными угла $\omega$-классическая область исследований в теории чисел, известная как неоднородные диофантовы приближения. Классическим результатом в этом направлении является теорема Минковского [6], которая утверждает, что если $t$ не лежит в орбите направления $\omega$, то неравенство $\|t+p \omega\|<1 /(4 p)$ имеет бесконечно много целочисленных решений $p$, причем постоянная $1 / 4$ в общем случае неулучшаема. Здесь $\|\cdot\|$ - стандартное расстояние на $\mathbb{S}^{1}$. B духе теоремы Минковского мы рассматриваем множество

$$
\left\{t \in \mathbb{S}^{1}:\|t+p \omega\|<p^{-\mu} \text { для бесконечно многих } p \in \mathbb{N}\right\} .
$$

Для каждого $\mu>1$ теорема 3.2 утверждает, что хаусдорфова размерность этого множества равна $\mu^{-1}$. Теорема 4.6 легко следует из аналогичного утверждения для аппроксимаций по арифметической прогрессии.

Другой классический результат теории чисел - теорема Ярника о хаусдорфовой размерности множества чисел, хорошо аппроксимируемых рациональными [7]. В духе теоремы Ярника мы рассмотрим множество

$$
\left\{\omega \in \mathbb{S}^{1}:\|t+p \omega\|<p^{-\mu} \text { для бесконечно многих } p \in \mathbb{N}\right\} .
$$

Левесли [8] доказал, что для любого $\mu>1$ хаусдорфова размерность этого множества равна $2 /(1+\mu)$. Доказательство теоремы 4.7 опирается на аналогичный неоднородный результат Ярника, обобшенный на арифметические подпоследовательности.

Структура работы такова. В $\S 2$ описываются необходимые результаты из теории размерности и теории бильярдов. Чисто теоретико-числовые результаты формулируются в $\S 3$, где также доказываются те из них, которые являются новыми. В $\S 4$ мы формулируем и доказываем новые результаты, касающиеся бильярдов. 
В заключение заметим, что результаты Сипры и др. были обобщены одним из нас в другом направлении [9].

\section{§ 2. Предварительные сведения}

2.1. Размерность. Пусть $Y$ - подмножество в $\mathbb{R}^{n}$. Пусть также $N(\epsilon)$ - минимальное число $\epsilon$-шаров, необходимое, чтобы покрыть $Y$.

ОПРЕДЕЛЕНИЕ 2.1. Нижняя bох-размерность $\operatorname{dim}_{L B} Y$ подмножества $Y \subset \mathbb{R}^{n}$ определяется формулой

$$
\operatorname{dim}_{L B} Y=\liminf _{\epsilon \rightarrow 0} \frac{\log N(\epsilon)}{\log 1 / \epsilon}
$$

Верхняя бох-размерность $\operatorname{dim}_{U}$ в определяется аналогично с заменой $\lim$ inf на limsup. Если и $\operatorname{dim}_{U} Y$ и $\operatorname{dim}_{L B} Y$ существуют и они совпадают, то bох-размерность подмножества $Y$ определяется как $\operatorname{dim}_{B} Y=\operatorname{dim}_{U} Y=\operatorname{dim}_{L B} Y$.

Через $\operatorname{diam} U$ будет обозначаться диаметр подмножества $U \subset \mathbb{R}^{n}$.

ОПРЕДЕЛЕНИЕ 2.2. Пусть $s \in[0, \infty]$. Тогда $s$-мерная мера Хаусдорфа $\mathscr{H}^{s}(Y)$ подмножества $Y \subset \mathbb{R}^{n}$ определяется как предел “є-обхватов":

$$
\mathscr{H}^{s}(Y)=\lim _{\epsilon \rightarrow 0}\left(\inf \left\{\sum_{i=1}^{\infty}\left(\operatorname{diam} U_{i}\right)^{s}: Y \subset \bigcup_{i=1}^{\infty} U_{i} \text { и } \sup _{i} \operatorname{diam} U_{i} \leqslant \epsilon\right\}\right) .
$$

Легко видеть, что существует и единственно такое число $s_{0}=s_{0}(Y)$, что

$$
\mathscr{H}^{s}(Y)=\left\{\begin{array}{l}
\infty \text { при } s<s_{0} \\
0 \quad \text { при } s>s_{0} .
\end{array}\right.
$$

ОПРЕДЕлЕНИЕ 2.3. Единственное число $s_{0}$, определяемое формулой $(1)$, называется хаусдорфовой размерностью множества $Y$ и обозначается через $\operatorname{dim}_{H} Y$.

Стандартные рассуждения показывают, что

$$
\operatorname{dim}_{H} Y \leqslant \operatorname{dim}_{L B} Y \leqslant \operatorname{dim}_{U B} Y
$$

для произвольного подмножества $Y$ в $\mathbb{R}^{n}$. Примеры показывают, что эти неравенства могут быть строгими.

Вох-размерность также может быть определена в терминах сумм, связанных с покрытиями, с единственным отличием, что все множества, образующие покрытие, должны иметь одинаковый диаметр. Заметим, что для того чтобы оценить box-размерность, достаточно потребовать, чтобы поперечники покрывающих множеств стремились к нулю по геометрической прогрессии.

Наконец, определим хаусдорфову размерность меры. 
ОПРЕДЕЛЕНИЕ 2.4. Пусть $\mu$-вероятностная борелевская мера на X. Хаусдорфова размерность меры $\mu$ определяется формулой

$$
\operatorname{dim}_{H} \mu=\inf _{Y}\left\{\operatorname{dim}_{H} Y: \mu(Y)=1\right\}
$$

Отметим, что размерность меры никогда не превосходит размерности ее (борелевского) носителя. Хорошо известен метод, позволяющий оценить снизу размерность меры или множества. Пусть $\mathscr{U}(x, r)$ - шар радиуса $r$ с центром в точке $x$.

Лемма 2.5. Пусть $\mu$-конечная мера. Если

$$
\liminf _{r \rightarrow 0} \frac{\log \mu(\mathscr{U}(x, r))}{\log r} \geqslant s
$$

на множестве полохсительной $\mu$-мерь, то размерность меры $\mu$ не меньие чем $s$.

Обзор методов и результатов теории размерности содержится в [10], [11].

2.2. Бильярдное преобразование направлений. Рассмотрим многоугольник $Q \subset \mathbb{R}^{2}$. Бильярдный шар, т.е. точечная масса, движется внутри $Q$ с единичной скоростью вдоль прямой до тех пор, пока не достигнет гранищы $\partial Q$, где он мгновенно меняет направление по закону "угол падения равен углу отражения" и продолжает движение по новой прямой. Предположим временно, что многоугольник $Q$ вьпуклый. Мы опишем бильярдное отображение в $Q$ как преобразование множества $X$ лучей, пересекающих $Q$.

Параметризуем $X$ двумя параметрами. В качестве первого параметра возьмем угол $\theta$ между лучом и положительной полуосью $x$. Чтобы определить второй параметр, рассмотрим перпендикулярное сечение $X_{\theta}$ множества лучей, имеющих угол $\theta$. Множество $X_{\theta}$ - это просто интервал, так что второй параметр это длина дуги на интервале $X_{\theta}$ с фиксированной ориентацией. Для невыпуклого многоугольника нужно различать части лучей, входящих в многоугольник и выходящих из него несколько раз. Можно осуществить указанную выше конструкцию локально, в результате чего получим множество $X_{\theta}$, представляющее собой конечное объединение интервалов. Пусть $w$ - (не обязательно нормированная) линейная мера Лебега на $X_{\theta}$.

Бильярдное отображение $T: X \rightarrow X$ переводит луч, содержащий отрезок бильярдной траектории, ориентированный направлением движения на нем, в луч, содержащий следующий отрезок траектории после отражения от границы.

Многоугольник $Q$ называется обобщенным параллелограммом, если каждая сторона его параллельна одному из двух заданных направлений. Предположим, что одно из этих направлений есть направление положительной полуоси $x$. Тогда существует единственное $\alpha$ в интервале $0<\alpha \leqslant \pi / 2$ такое, что угол между любыми двумя сторонами равен $0, \alpha$ или $\pi-\alpha$. Всюду в работе предполагается, что $\alpha \notin \mathbb{Q} \cdot \pi$, т.е. $Q$ не является рациональным многоугольником. Заметим, что бильярд в прямоугольном треугольнике эквивалентен бильярду в ромбе, полученном из четырех экземпляров этого треугольника с помощью процедуры развертки. 
Бильярдное отображение в обобшенном параллелограмме имеет следующие специальњые свойства (см. рис. 1). Множество $X_{\theta}$ может быть (локально) разбито на три множества $U_{\theta}, R_{\theta}$ и $D_{\theta}$, состоящие из конечного объединения интервалов, такие, что $T^{2} U_{\theta} \subset X_{\theta+2 \alpha}, T^{2} R_{\theta} \subset X_{\theta}, T^{2} D_{\theta} \subset X_{\theta-2 \alpha}$ и каждый интервал в $U_{\theta}, R_{\theta}$ и $D_{\theta}$ изометрически отображается на свой образ. Линейная мера $w$ $T$-инвариантна. Далее, существует такая постоянная $K$, что

$$
w\left(D_{\theta}\right) \leqslant K|\sin \theta| \text { и } w\left(U_{\theta}\right) \leqslant K|\sin (\theta-\alpha)| .
$$

Концы интервалов, составляющих $U_{\theta}$ и $D_{\theta}$, будут называться особенностями отображения $T^{2}$.

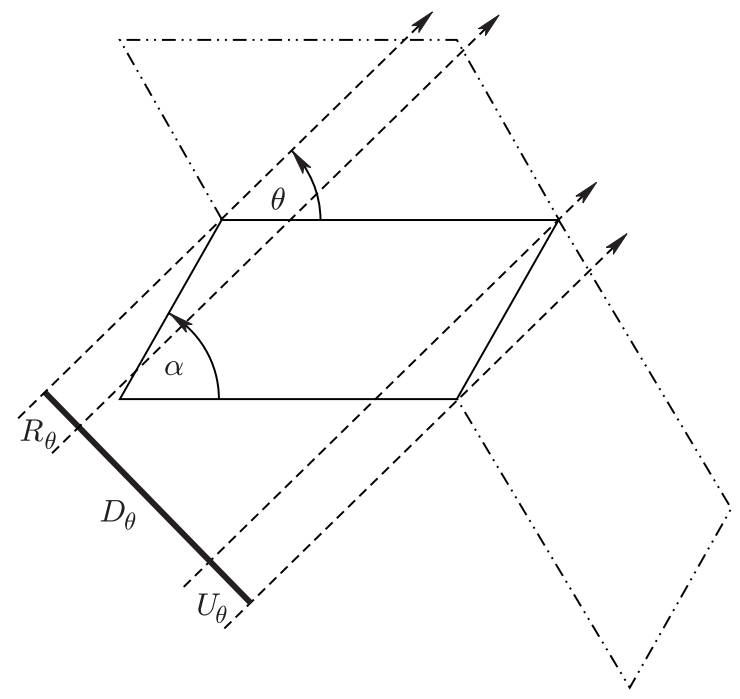

Обозначим $Z_{n}:=X_{\theta+2 n \alpha}$ и $Z:=\bigcup_{n \in \mathbb{Z}} Z_{n}$. Множество $Z_{n}$ называется $n$-м уровнем множества $Z$. Множество $Z T^{2}$-инвариантно с бесконечной инвариантной мерой $w$.

О бильярдах написано несколько хороших обзорных статей [12]-[14]. Обсуждаемая здесь структура более подробно описана в [5].

\section{§3. Теоретико-числовые результаты}

В этом параграфе мы работаем с окружностью $\mathbb{R} / \mathbb{Z}$ единичной длины, а не длины $2 \pi$. Роль углов $\theta$ и $\alpha$, рассматриваемых в параграфах, посвященных бильярдам, играют здесь параметры $t=(\theta \bmod \pi) / \pi$ и $\omega=(2 \alpha \bmod \pi) / \pi$.

3.1. Неоднородные диофантовы приближения. Рассмотрим множество

$$
\mathscr{A}_{\mu, \omega}:=\left\{t:\|t+p \omega\|<|p|^{-\mu}\right. \text { для бесконечного набора положительных }
$$

и бесконечного набора отрицательных $p \in \mathbb{Z}\}$.

Пусть $m, l \in \mathbb{N}$ - заданные числа такие, что $0 \leqslant l<m$. Положим

$$
\begin{array}{r}
\mathscr{A}_{\mu, \omega}(l, m):=\left\{t:\|t+p \omega\|<|p|^{-\mu}\right. \text { для бесконечного набора положительных } \\
\text { и бесконечного набора отрицательных } p \equiv l \bmod m\} .
\end{array}
$$

Заметим, что $\mathscr{A}_{\mu, \omega}(l, m) \subset \mathscr{A}_{\mu, \omega}$ и $\mathscr{A}_{\mu, \omega}=\bigcup_{0 \leqslant l<m} \mathscr{A}_{\mu, \omega}(l, m)$. 
Минковский показал, что если $t$ не лежит в орбите числа $\omega$, то неравенство $\|t+p \omega\|<1 /(4 p)$ имеет бесконечно много решений, причем постоянная $1 / 4$ в обшем случае оптимальна [6]. Простое рассуждение типа Бореля-Кантелли показывает, что множество направлений, аппроксимируемых лучше, чем указано в теореме Минковского, имеет меру нуль.

ПрЕДЛОЖЕНИЕ 3.1. Пусть $\mu>1 u \omega \notin \mathbb{Q}$. Мера Лебега множества $\mathscr{A}_{\mu, \omega}$ равна нулю, при этом $\operatorname{dim}_{B} \bigcap_{l=0}^{m-1} \mathscr{A}_{\mu, \omega}(l, m)=1$. Множества $\mathscr{A}_{\mu, \omega}(l, m)$ остаточные. Поэтому множество $\bigcap_{\mu \in \mathbb{Q}, \mu>1} \bigcap_{l=0}^{m-1} \mathscr{A}_{\mu, \omega}(l, m)-$ также остаточное множество и его бох-размерность равна единице.

ДокАЗАТЕльство. Для простоты при $0<a<b$ мы обозначаем интервал $(-b,-a)$ через $-(a, b)$. Пусть $S(l, m)=\{n \in \mathbb{Z}: n \equiv l \bmod m\}$. Тогда

$$
\mathscr{A}_{\mu, \omega}(l, m)=\bigcap_{|k|=1}^{\infty} \bigcup_{p \in S(l, m) \cap \operatorname{sgn}(k)[|k| m, \infty)}\left(p \omega-\frac{1}{2|p|^{\mu}}, p \omega+\frac{1}{2|p|^{\mu}}\right)
$$

$$
\mathscr{A}_{\mu, \omega}=\bigcap_{|k|=1}^{\infty} \bigcup_{p \in \operatorname{sgn}(k)[|k|, \infty)}\left(p \omega-\frac{1}{2|p|^{\mu}}, p \omega+\frac{1}{2|p|^{\mu}}\right) .
$$

Поскольку множества $\{p \omega: p \in S(l, m) \cap[|k| m, \infty)\}$ и $\{p \omega: p \in S(l, m) \cap$ - $[|k| m, \infty)\}$ плотны, то $\mathscr{A}_{\mu, \omega}(l, m)$ - остаточное множество. Итак, множество $\bigcap_{k=0}^{m-1} \mathscr{A}_{\mu, \omega}(l, m)$ плотно, а значит, его верхняя и нижняя bох-размерности равны единице. Чтобы убедиться, что лебегова мера множества $\mathscr{A}_{\mu, \omega}$ равна нулю, заметим, что последовательность $\left(2|p|^{\mu}\right)_{p \in \mathbb{Z}}^{-1}$ имеет при $\mu>1$ конечную сумму. По лемме Бореля-Кантелли почти никакая точка $t$ не содержится более чем в конечном числе интервалов $\left(p \omega-1 /\left(2|p|^{\mu}\right), p \omega+1 /\left(2|p|^{\mu}\right)\right)$.

Используя это предложение, рассмотрим размерность множества $\mathscr{A}_{\mu, \omega}$ точек $t$, обладающих лучшими аппроксимациями.

Теорема 3.2. Для любъх $\mu>1 u m \in \mathbb{N}$

$$
\operatorname{dim}_{H} \bigcap_{l=0}^{m-1} \mathscr{A}_{\mu, \omega}(l, m)=\frac{1}{\mu}
$$

ДокаЗАТЕльство. Докажем сперва оценку сверху. Для любого $\varepsilon>0$ из формулы (3) вытекает, что

$$
\mathscr{H}^{\frac{1}{\mu}+\varepsilon}\left(\mathscr{A}_{\mu, \omega}(l, m)\right) \leqslant 2 \cdot \liminf _{k \rightarrow \infty} \sum_{p=k}^{\infty}\left(\frac{1}{p^{\mu}}\right)^{\frac{1}{\mu}+\varepsilon}=0 .
$$

Обратимся теперь к оценке снизу. При $n_{k} \in \mathbb{Z}$ положим $\mathscr{J}_{k}:=\operatorname{sgn}\left(n_{k}\right)\left[\left|n_{k}\right|, 2\left|n_{k}\right|\right]$ и

$$
\widehat{T}_{n_{k}}^{\mu}:=\bigcup_{q m+k \in \mathscr{J}_{k}}\left((q m+k) \omega-\frac{1}{2\left(2\left|n_{k}\right|\right)^{\mu}},(q m+k) \omega+\frac{1}{2\left(2\left|n_{k}\right|\right)^{\mu}}\right) .
$$


Для произвольной последовательности цельх чисел $\left\{n_{k}\right\}$, как положительные, так и отрицательные элементы $n_{k}$ которой бесконечно часто попадают во все классы вычетов, верно, что

$$
\widehat{S}_{\left\{n_{k}\right\}}:=\bigcap_{k=1}^{\infty} \widehat{T}_{n_{k}}^{\mu} \subset \bigcap_{l=0}^{m-1} \mathscr{A}_{\mu, \omega}(l, m) .
$$

Для доказательства оценки снизу мы построим по индукции последовательность $\left\{n_{k}\right\}$, которая будет просто подпоследовательностью последовательности знаменателей $q_{m}$ подходящих дробей непрерывной дроби. Для этой последовательности $\left\{n_{k}\right\}$ мы построим меру $m$ с носителем на множестве $\widehat{S}_{\left\{n_{k}\right\}}$ размерности $\mu^{-1}$. Идея построения заключается в следующем: чем быстрее возрастает последовательность $\left\{n_{k}\right\}$, тем лучше точки $((q m+k) \omega)_{q m+k \in \mathscr{J}_{k}}$ распределены на окружности. Если точка $x$ лежит в $\mathscr{A}_{\mu, \omega}$, то она должна бесконечно часто встречаться на интервалах вида $J_{q}=\left(q \omega-|q|^{-\mu} / 2, q \omega+|q|^{-\mu} / 2\right)$. Поэтому для некоторого достаточно большого $n_{k}$ она неизбежно попадет в $J_{q}$ для некоторого $q \in \operatorname{sgn}\left(n_{k}\right)\left[0,2\left|n_{k}\right|\right]$. Принадлежность множеству $\widehat{S}_{\left\{n_{k}\right\}}$ вынуждает эту точку находиться в “правой половине" семейства интервалов $J_{q}$, где $q$ меняется от 0 до $2 n_{k}$. Если последовательность $\left\{n_{k}\right\}$ достаточно разрежена, то это ограничение оказывается достаточно умеренньм для сохранения размерности множества $\mathscr{A}_{\mu, \omega}$.

Положим $n_{1}=q_{1}$ и предположим, что последовательность $n_{i}$ уже построена вплоть до $i=k-1$. Тогда множество $\bigcap_{l=1}^{k-1} \widehat{T}_{n_{l}}^{\mu}$ состоит из конечного числа открытых интервалов $\widehat{I}_{i}^{k-1}$, удовлетворяющих неравенству

$$
\left|\widehat{I}_{l}^{k-1}\right| \leqslant\left(2\left|n_{k-1}\right|\right)^{-\mu}
$$

в котором равенство выполняется тогда и только тогда, когда соответствующий интервал полностью содержится в $\bigcap_{l=1}^{k-2} \widehat{T}_{n_{l}}^{\mu}$. Мы будем опускать крышку в обозначении любого интервала, для которого выполнено равенство.

Рассмотрим множество

$$
T_{n_{k}}^{\mu}:=\bigcup_{l} I_{l}^{k}
$$

и положим

$$
S_{\left\{n_{k}\right\}}:=\bigcap_{k=1}^{\infty} T_{n_{k}}^{\mu} .
$$

Очевидно, $S_{\left\{n_{k}\right\}} \subset \widehat{S}_{\left\{n_{k}\right\}}$.

Предположим, что $\left\{n_{i}\right\}$ уже построены по индукции вплоть до $i \leqslant k-1$. Хорошо известно, что для любого $k \in \mathbb{N}$ последовательность $\{(p m+k) \omega: p \in \mathbb{Z}\}$ "хорошо распределена" на $\mathbb{S}^{1}$, т.е. для любой непрерывной функции $f$ и любого $\varepsilon>0$ сушествует такое число $N$, что

$$
\left|\frac{1}{n} \sum_{p=0}^{n-1} f((q+p m+k) \omega)-\int f\right|<\varepsilon
$$


для любого $q \in \mathbb{Z}$ и любого $n \geqslant N$. Поэтому при $k(\bmod 2 m) \leqslant m-1$ в качестве $n_{k}$ можно взять наименьшее целое положительное $q_{r}>\left|n_{k-1}\right|$, удовлетворяющее следующим двум условиям:

$$
\frac{\left|I_{l}^{k-1}\right|}{2} \leqslant \frac{\operatorname{Card}\left\{p m+k \in \mathscr{J}_{k}:(p m+k) \omega \in I_{l}^{k-1}\right\}}{\left|q_{r}\right|} \leqslant 2\left|I_{l}^{k-1}\right|
$$

$$
\frac{\log \prod_{i=1}^{k-1}\left|n_{i}\right|}{\log \left|q_{r}\right|} \rightarrow 0
$$

В противном случае (при $k(\bmod 2 m) \geqslant m)$ мы полагаем $n_{k}=-q_{r}$, удовлетворяя условиям (5) и (4). Построение последовательности $\left\{n_{k}\right\}$ завершено. Очевидно, эта последовательность попадает во все классы вычетов бесконечно часто как для положительных, так и для отрицательных $n_{k}$.

Далее на $S_{\left\{n_{k}\right\}}$ построим меру нужной размерности. Мы предполагаем, что $I^{0}$ совпадает со всей окружностью и потому имеет длину 1. Сначала рекуррентно определим внешнюю меру $m^{*}$, полагая $m^{*}\left(I^{0}\right):=1$ и

$$
m^{*}\left(I_{l}^{k}\right):=\frac{m^{*}\left(I_{j}^{k-1}\right)}{\operatorname{Card}\left\{j: I_{l}^{k} \subset I_{j}^{k-1}\right\}} .
$$

Теперь мы оценим внешнюю меру сверху. Используя формулы (5) и (6), имеем

$$
\begin{aligned}
m^{*}\left(I_{j}^{k+1}\right) & \leqslant \frac{2 m^{*}\left(I_{l}^{k}\right)}{\left|I_{l}^{k}\right|\left|n_{k+1}\right|}=2 m^{*}\left(I_{l}^{k}\right) \frac{\left(2\left|n_{k}\right|\right)^{\mu}}{\left|n_{k+1}\right|} \\
& \leqslant 2^{(1+\mu) k} \prod_{i=1}^{k} \frac{\left(\left|n_{i}\right|\right)^{\mu}}{\left|n_{i+1}\right|} \leqslant 2^{(1+\mu) k} \frac{\left(n_{1}\right)^{\mu}}{\left|n_{k+1}\right|} \prod_{i=2}^{k}\left(\left|n_{i}\right|\right)^{\mu-1} .
\end{aligned}
$$

Большая скорость роста чисел $n_{k}$, предполагаемая в (6), влечет соотношение $m^{*}\left(I_{j}^{k}\right) \rightarrow 0$ при $k \rightarrow \infty$. Поэтому ясно, что $m^{*}$ удовлетворяет условиям согласованности Колмогорова и может быть продолжена до меры $m$ на $S_{\left\{n_{k}\right\}}$.

Теперь мы можем доказать, что размерность этой меры не меньше чем $1 / \mu$. Поскольку $\left|I^{k+1}\right|=\left(2\left|n_{k-1}\right|\right)^{-\mu}<1$, то из (7) вытекает, что

$$
\begin{aligned}
\frac{\log m\left(I_{l}^{k+1}\right)}{\log \left|I_{l}^{k+1}\right|} & \geqslant \frac{\log 2^{(1+\mu) k}+\sum_{i=2}^{k} \log \left|n_{i}\right|^{\mu-1}+\mu \log n_{1}-\log \left|n_{k+1}\right|}{\log \left(2\left|n_{k+1}\right|\right)^{-\mu}} \\
& =\frac{-k C_{1}-C_{2} \sum_{i=2}^{k} \log \left|n_{i}\right|-C_{3}+\log \left|n_{k+1}\right|}{C_{4}+\mu \log \left|n_{k+1}\right|},
\end{aligned}
$$

где все $C_{i}$ - положительные постоянные. Согласно (6) имеем

$$
\lim _{k \rightarrow \infty} \frac{\log m\left(I_{l}^{k+1}\right)}{\log \left|I_{l}^{k+1}\right|} \geqslant \frac{1}{\mu}
$$


Чтобы применить лемму 2.5 и вывести требуемый результат, нужно вычислить отношение $\log m(I) / \log |I|$ для "промежуточных" интервалов $I$, т.е. интервалов, удовлетворяющих неравенствам

$$
\frac{1}{\left(2\left|n_{k}\right|\right)^{\mu}}<|I|<\frac{1}{\left(2\left|n_{k-1}\right|\right)^{\mu}} .
$$

Обозначим через $\mathscr{U}_{a}(B) a$-окрестность множества $B$ и положим

$$
r(I):=\operatorname{Card}\left\{q m+k: q m+k \in \mathscr{J}_{k} \text { и }(q m+k) \omega \in I \backslash \mathscr{U}_{\left(2 n_{k}\right)-\mu}(\partial I)\right\} .
$$

Для $r=1$ обозначим через $I_{l}^{k}$ единственный такой интервал, содержащийся в $I$. Тогда ввиду соотношений $m\left(I_{l}^{k}\right)=m(I)$ и $\left|I_{l}^{k}\right|<|I|$ имеем

$$
\frac{\log m(I)}{\log |I|}=\frac{\log m\left(I_{l}^{k}\right)}{\log |I|}>\frac{\log m\left(I_{l}^{k}\right)}{\log \left|I_{l}^{k}\right|} .
$$

Предположим теперь, что $r \geqslant 2$. Поскольку $\left|n_{k}\right|$ - это знаменатель $q_{m_{k}}$ некоторой подходящей дроби, то

$$
\min _{\left|n_{k}\right| \leqslant\left|p_{1}\right|<\left|p_{2}\right| \leqslant\left|2 n_{k}\right|} d\left(p_{1} \omega, p_{2} \omega\right) \geqslant \frac{1}{\left|n_{k}\right|+2} .
$$

Отметим, что это единственное место, где используется тот факт, что $n_{k}-$ подпоследовательность последовательности знаменателей подходящих дробей непрерывной дроби. Из (8) и (4) (в предположении, что $\left|n_{k}\right| \geqslant 2$ ) вытекает, что

$$
|I| \geqslant(r-1) \frac{1}{\left|n_{k}\right|+2} \geqslant(r-1)\left|I_{l}^{k}\right|^{\frac{1}{\mu}} .
$$

Поэтому справедлива оценка

$$
\begin{aligned}
\frac{\log m(I)}{\log |I|} & \geqslant \frac{\log m(I)}{\log \left((r-1) \cdot\left|I_{l}^{k}\right|^{\frac{1}{\mu}}\right)} \\
& \geqslant \frac{\log \left(r \cdot m\left(I_{l}^{n}\right)\right)}{\log \left((r-1) \cdot\left|I_{l}^{k}\right|^{\frac{1}{\mu}}\right)} \\
& =\frac{\log r+\log m\left(I_{l}^{k}\right)}{\log (r-1)+\frac{1}{\mu} \log \left|I_{l}^{k}\right|}
\end{aligned}
$$

Для любого $\varepsilon>0$, используя неравенство (9), мы можем выбрать достаточно большое $k(\varepsilon)$, так что

$$
\frac{\log m(I)}{\log |I|} \geqslant \frac{\log r+\frac{1}{\mu+\varepsilon} \log \left|I_{l}^{k}\right|}{\log (r-1)+\frac{1}{\mu} \log \left|I_{l}^{k}\right|}
$$

для любого $k \geqslant k(\varepsilon)$. Поэтому

$$
\frac{\log m(I)}{\log |I|} \geqslant \frac{\mu}{\mu+\varepsilon}>\frac{1}{\mu}
$$

где последнее неравенство выполняется для любого достаточно малого $\varepsilon$.

Теперь наш результат следует из леммы 2.5. 


\section{2. Теорема Ярника. Рассмотрим множество}

$$
\begin{gathered}
\mathscr{B}_{\mu, t}:=\left\{\omega:\|t+p \omega\|<|p|^{-\mu}\right. \text { для бесконечного набора положительных } \\
\text { и бесконечного набора отрицательных } p \in \mathbb{Z}\} .
\end{gathered}
$$

Пусть $m, l \in \mathbb{N}$ - заданные числа такие, что $0 \leqslant l<m$, и пусть

$$
\begin{array}{r}
\mathscr{B}_{\mu, t}(l, m):=\left\{\omega:\|t+p \omega\|<|p|^{-\mu}\right. \text { для бесконечного набора положительных } \\
\text { и бесконечного набора отрицательных } p \equiv l \bmod m\} .
\end{array}
$$

Тогда $\mathscr{B}_{\mu, t}(l, m) \subset \mathscr{B}_{\mu, t}$ и $\mathscr{B}_{\mu, t} \supset \bigcup_{0 \leqslant l<m} \mathscr{B}_{\mu, t}(l, m)$.

ПреДЛОЖЕНИЕ 3.3. Предположим, ито $\mu>1 u \omega \notin \mathbb{Q}$. Тогда мера Лебега мнохсества $\mathscr{B}_{\mu, t}(l, m)$ равна нулю, а $\operatorname{dim}_{B} \mathscr{B}_{\mu, t}(l, m)=1$. Множества $\mathscr{B}_{\mu, t}(l, m)$ остаточные.. Поэтому множество $\bigcap_{\mu \in \mathbb{Q}, \mu>1} \bigcap_{0 \leqslant l<m} \mathscr{B}_{\mu, t}(l, m)$ такхе остаточное и имеет бох-размерность 1.

ДОкаЗАТЕЛЬСТво. Пусть $S(l, m)=\{n \in \mathbb{N}: n \equiv l \bmod m\}$, тогда

$$
\mathscr{B}_{\mu, t}(l, m)=\bigcap_{|k|=1}^{\infty} \bigcup_{p \in S(l, m) \cap \operatorname{sgn}(k)[|k|, \infty)} \bigcup_{i=0}^{p-1}\left(\frac{t+i}{p}-\frac{1}{2|p|^{\mu+1}}, \frac{t+i}{p}+\frac{1}{2|p|^{\mu+1}}\right)
$$

и поэтому $\mathscr{B}_{\mu, t}(l, m)$ - остаточное множество. Множество $\mathscr{B}_{\mu, t}(l, m)$ плотно, так что его нижняя и верхняя bох-размерности равны единице. Поскольку $\sum 1 /\left(2|p|^{\mu+1}\right)<\infty$, по лемме Бореля-Кантелли почти ни одна точка $\omega$ не содержится более чем в конечном числе интервалов $\left(\frac{t+i}{p}-\frac{1}{2||^{\mu+1}}, \frac{t+i}{p}+\right.$ $\left.\frac{1}{2|p|^{\mu+1}}\right)$, т.е. лебегова мера множества $\mathscr{B}_{\mu, t}(l, m)$ равна нулю.

Следующее ниже обобщение классической теоремы Ярника [7] представляет собой частный случай еще более общего результата, доказанного Левесли.

Теорема 3.4 [8]. Для любого $\mu>1$ имеем

$$
\operatorname{dim}_{H} \mathscr{B}_{\mu, t}=\frac{2}{1+\mu} .
$$

Мы улучшим теорему Левесли в этой специальной постановке. Доказательство по сушеству следует его идеям и не претендует на оригинальность.

Теорема 3.5. Для любъх $\mu>1 u m \in \mathbb{N}$ имеем

$$
\operatorname{dim}_{H} \bigcap_{l=0}^{m-1} \mathscr{B}_{\mu, t}(l, m)=\frac{2}{1+\mu} .
$$

Нам потребуется приспособленное к нашему случаю понятие вездесущей системы. Для заданных $l$ и $m$ определим множество

$$
R_{k}(l, m)=\{t \in[0,1):\|(k m+l) t-\omega\|=0\} .
$$


ОПРЕДЕЛЕНИЕ 3.6. Пусть $\rho: \mathbb{N} \rightarrow \mathbb{R}$ - такая функция, что $\lim _{N \rightarrow \infty} \rho(N)=0$. Семейство множеств $\left\{R_{k}(l, m)\right\}$ называется вездесущим относительно $\rho$, если

$$
\lim _{N \rightarrow \infty} \operatorname{mes}\left([0,1) \backslash \bigcup_{k=1}^{N} \mathscr{U}\left(R_{k}(l, m), \rho(N)\right)\right)=0
$$

Множества $\mathscr{B} \mu, t(l, m)$ представляются в виде

$$
\begin{aligned}
& \mathscr{B}_{\mu, t}^{+}(l, m):=\bigcap_{K=0}^{\infty} \bigcup_{k=K}^{\infty} \mathscr{U}\left(R_{k}(l, m),(k m+l)^{-\mu-1}\right), \\
& \mathscr{B}_{\mu, t}^{-}(l, m):=\bigcap_{K=0}^{\infty} \bigcup_{-k=K}^{\infty} \mathscr{U}\left(R_{k}(l, m),(k m+l)^{-\mu-1}\right)
\end{aligned}
$$

и

$$
\mathscr{B}_{\mu, t}(l, m)=\mathscr{B}_{\mu, t}^{+}(l, m) \cap \mathscr{B}_{\mu, t}^{-}(l, m) .
$$

Мы используем следующий частньй случай более общей теоремы 2 из статьи Додсона, Рюнне и Викерса [15].

ТЕОРема 3.7 [15]. Предположсим, что для любого $l$ в интервале $0 \leqslant l<m$ семейство $\left\{R_{k}(l, m)\right\}$ - вездесущее относительно $\rho$. Тогда

$$
\operatorname{dim}_{H} \bigcap_{l=0}^{m-1} \mathscr{B}_{\mu, t}(l, m) \geqslant \min \left\{1, \limsup _{N \rightarrow \infty} \frac{\log \rho(N)}{\mu+1}\right\}
$$

Левесли доказал, что система $\left\{\bigcup_{l=0}^{m-1} R_{k}(l, m)\right\}$ - вездесущая относительно функции $\rho(N):=K \frac{\log ^{5+\varepsilon} N}{N^{2}}$. Мы можем воспроизвести рассуждение Левесли и показать, что $\left\{R_{k}(l, m)\right\}$ - вездесушая система относительно функции $\rho(N):=$ $K \frac{\log ^{5+2 \varepsilon}(N m+l)}{(N m+l)^{2}}$. Теперь теорема 3.5 вытекает из теоремы 3.7 .

\section{§4. Результаты о бильярдах}

4.1. Сильная возвращаемость во всех направлениях. Зафиксируем обобшенньй параллелограмм. Для каждого направления $\theta$ обозначим через $F_{\theta}$ множество элементов $x \in X_{\theta}$, положительная полутраектория которых никогда не возвращается параллельно $x$, и положим $G_{\theta}:=X_{\theta} \backslash F_{\theta}$.

ТЕОРема 4.1. Для любого обобщенного параллелограмма и любого направления $\theta$ нижняя бох-размерность мнохества $F_{\theta}$ не превышает 1/2.

Это утверждение усиливает основную теорему в [5], которая утверждает, что для любого $\theta$ множество $F_{\theta}$ имеет меру 0 . Заметим, что множество $G_{\theta}$ открыто и плотно для каждого $\theta$.

Развертка прямоугольного треугольника с углом $\alpha / 2$ вокруг прямого угла дает ромб с углом $\alpha$. Рассмотрим в ромбе направление $\theta$, перпендикулярное одному 
из катетов треугольника. Если рассматривать траектории, начинающиеся в $X_{\theta}$ и возврашаюшиеся в $X_{\theta}$, как траектории в треугольнике, то они оказываются дважды перпендикулярными одной из сторон. Как объяснено во введении, такие траектории должны быть периодическими. Поэтому, применяя теорему 4.1 к этому направлению, получаем следующее усиление теоремы Сипры, Хансона и Колана [4]:

СлЕДСТВИЕ 4.2. В прямоугольном треугольнике ниэняя фрактальная размерность множества элементов, перпендикулярных одному из катетов треугольника, для которьх траектория не является периодической, не превыниает $1 / 2$.

В доказательстве теоремы 4.1 нам понадобится лемма, которая, по существу, содержится в [5].

Лемма 4.3. Зафиксируем интервал $X_{\theta}$ и натуральное $N$. Тогда существует разбиение множсества $U_{\theta}$ на $j_{N} \leqslant C N$ интервалов (әде $C$-некоторая положительная постоянная) такое, что итерации $T^{2}$ отображсают изометрично каждый интервал разбиения до тех пор, пока не встретится одна из следующих ситуаций: 1) траектория интервала достигает уровня $N$ прежсде, чем вернуться на $X_{\theta}$, или 2) траектория интервала возвращается на $X_{\theta}$, так и не достигнув уровня $N$. Траектории всех этих интервалов попарно не пересекаются до возмознного момента возвращения на $X_{\theta}$.

Аналогичное утверждение верно для $D_{\theta}$ и уровня $-M$.

ДокАЗАтЕльство. Рассмотрим множество $V_{N}$ особенностей отображения $T^{2}$ на уровнях $0,1, \ldots, N$. Мошность множества $V_{N}$ не превосходит $C N$ (где в качестве $C$ можно взять удвоенное число вершин многоугольника). Для каждого $v \in V_{N}$ рассмотрим его первый прообраз, лежащий на уровне 0 (если таковой существует: заметим, что время, необходимое для того, чтобы отобразить точку $v \in V_{N}$ на уровень 0 , не обязательно ограничено). Это дает указанное разбиение множества $U_{\theta}$. Отображение $T^{2 j}$ непрерьвно на каждом элементе разбиения до тех пор, пока он не отображается на уровень $N+1$ или не возврашается на уровень 0 .

Тот факт, что траектории не пересекаются, следует из обратимости отображения $T^{2}$. Действительно, предположим, что $x, y \in X_{\theta}, x \neq y$, но $z=T^{2 j} x=T^{2 k} y$ при некоторых $j \geqslant k$. Равенство $j=k$ невозможно, поскольку в этом случае точка $z$ имела бы два прообраза. Так как $T^{2(j-k)} x=y$, то траектория точки $x$ возвращается на $X_{\theta}$ в момент времени $2(j-k)>0$ и не пересекается с траекторией точки $y$ до этого времени, как и утверждалось.

Если бы один из интервалов (скажем, $J$ ) никогда не вернулся на $X_{\theta}$ и не достиг уровня $N$, то множество $\bigcup_{j \in \mathbb{N}} T^{2 j} J$ состояло бы из бесконечного числа непересекающихся интервалов одинаковой длины и содержалось бы в $\bigcup_{0 \leqslant n \leqslant N} Z_{n}$. Последнее множество есть конечное объединение интервалов конечной длины, так что мы приходим к противоречию.

ДоКАЗАТЕЛЬСТво ТЕОРЕмы 4.1. Зафиксируем произвольное $\theta$. Мы изучим размерность пересечения $F_{\theta} \cap D_{\theta}$. Рассуждение для $F_{\theta} \cap U_{\theta}$ аналогично, с дополнительным условием, что поскольку мы пользуемся формулой (2), то $\theta-2 N \alpha$ должно быть близко к нулю в случае $D_{\theta}$, в то время как в случае $U_{\theta}$ число $\theta+(2 N-1) \alpha$ 
должно быть близко к $\alpha$. В силу этого дополнительного условия и поскольку требуется, чтобы $F_{\theta} \cap D_{\theta}$ и $F_{\theta} \cap U_{\theta}$ были малы одновременно, вместо аппроксимаций с помощью $2 \alpha$ мы рассмотрим аппроксимации с помощью $\alpha$, используя четные итерации для множества $F_{\theta} \cap D_{\theta}$ и нечетные итерации для множества $F_{\theta} \cap U_{\theta}$.

Рассмотрим множество $F_{N}$ точек $x \in D_{\theta}$, положительная полутраектория которых достигает $X_{\theta-2(N+1) \alpha}$ прежде, чем вернуться на $X_{\theta}$. Это событие назовем выходом точки $x$ с уровня $-N$ (обязательно на уровень $-(N+1))$. Когда траектория точки $x$ достигает уровня $-N$ и находится в процессе выхода с уровня $-N$, она должна пройти через “ворота" $D_{\theta-2 N \alpha}$ на уровне $-N$.

Из леммы 4.3 ясно, что не более $C N$ интервалов выходит с уровня $-N$ прежде, чем вернуться на $X_{\theta}$. Поэтому множество $F_{N}$ состоит из не более чем $C N$ интервалов, которые мы обозначим через $I_{i}$. Когда они выходят с уровня $-N$ прежде, чем вернуться на уровень 0, сумма их длин не может превосходить ширину ворот на уровне $-N$, через которые они выходят. Подчеркнем, что здесь мы пользуемся утверждением из леммы 4.3 о том, что все выходящие траектории не пересекаются. Таким образом, суммарная длина интервалов, выходящих с уровня $-N$, не превосходит общей длины ворот уровня $-N$, т.е. не превосходит $K|\sin (\theta-2 N \alpha)|$.

Через $[x]$ мы обозначаем целую часть числа $x$. Пусть $\left\{a_{i}\right\}$ - последовательность положительных чисел, а $n \in \mathbb{N}$. Простая оценка показывает, что (см. [16])

$$
\sum_{k=1}^{n}\left(\left[\frac{n a_{k}}{\sum_{i=1}^{n} a_{i}}\right]+1\right) \leqslant 3 n .
$$

Можно оценить $(1 / 2+\varepsilon)$-обхват $F_{\theta} \cap D_{\theta}$, покрывая $F_{N}$ интервалами “средней выходной длины", т.е. интервалами длины $K|\sin (\theta-2 N \alpha)| / j_{N}$.

Необходимое для этого общее число интервалов средней длины не превосходит

$$
\sum_{k=1}^{j_{N}}\left(\left[\frac{\left|I_{k}\right| j_{N}}{\sum_{i=1}^{j_{N}}\left|I_{i}\right|}\right]+1\right) .
$$

Используя неравенство (11) и тот факт, что $j_{N} \leqslant C N$, получаем

$$
\begin{aligned}
\mathscr{H}^{1 / 2+\varepsilon}\left(F_{\theta} \cap D_{\theta}\right) & \leqslant \sum_{k=1}^{j_{N}}\left(\left[\frac{\left|I_{k}\right| j_{N}}{\sum_{i=1}^{j_{N}}\left|I_{i}\right|}\right]+1\right)\left(\frac{K(|\sin (\theta-2 N \alpha)|)}{j_{N}}\right)^{1 / 2+\varepsilon} \\
& \leqslant \frac{3 j_{N}}{\left(j_{N}\right)^{1 / 2+\varepsilon}}(K(|\sin (\theta-2 N \alpha)|))^{1 / 2+\varepsilon} \\
& \leqslant L N^{1 / 2-\varepsilon}((\theta-2 N \alpha) \bmod \pi)^{1 / 2+\varepsilon}
\end{aligned}
$$

с некоторой постоянной $L$. Чтобы получить требуемое покрытие, нужно только найти такую последовательность $\left(N_{k}\right)$, что неоднородное диофантово приближение $\left\|\left(\theta-2 N_{k} \alpha\right) / \pi\right\|$ мало́. Хорошо известно, что для любого $\delta>0$ неравенство

$$
\|(\theta-2 p \alpha) / \pi\|<p^{-1+\delta}
$$


разрешимо для бесконечно многих $p \in \mathbb{N}$. Заметим, что это гораздо более слабое утверждение, чем теорема Минковского [6]. Поэтому можно найти такие последовательности $\left(N_{k}\right)_{k \in \mathbb{N}}$, что

$$
\mathscr{H}^{1 / 2+\varepsilon}\left(F_{\theta} \cap D_{\theta}\right) \leqslant L N_{k}^{1 / 2-\varepsilon}\left(N_{k}^{-1+\delta}\right)^{1 / 2+\varepsilon} \leqslant L N_{k}^{-2 \varepsilon+(\delta / 2)+\delta \varepsilon} .
$$

Если $1>\varepsilon>\delta>0$, то правая часть стремится к нулю при $k \rightarrow \infty$.

Поскольку мы покрыли $F_{\theta} \cap D_{\theta}$ интервалами одинаковой длины, то

$$
\operatorname{dim}_{L B}\left(F_{\theta} \cap D_{\theta}\right) \leqslant \frac{1}{2}
$$

Чтобы оценить размерность множества $F_{\theta} \cap U_{\theta}$, в формуле $(12)$ нужно воспользоваться второй частью формулы (2). Имея в виду это изменение, полезно рассмотреть переменную $\theta^{\prime}:=\theta-\alpha$. Замечая, что $\theta+2 p \alpha-\alpha=\theta^{\prime}+2 p \alpha$ и неравенство $\left\|\left(\theta^{\prime}+2 p \alpha\right) / \pi\right\|<p^{-1+\delta}$ имеет бесконечно много целочисленных решений, мы приходим к выводу, что $\operatorname{dim}_{L B}\left(F_{\theta} \cap U_{\theta}\right) \leqslant 1 / 2$ и поэтому $\operatorname{dim}_{L}\left(F_{\theta}\right) \leqslant 1 / 2$.

4.2. Из теории чисел вытекает более сильная возвращаемость в некоторых направлениях. В предыдущем пункте мы доказали теорему о возвращаемости для всех направлений $\theta$. Наши первые результаты в этом пункте относятся к специальным направлениям $\theta$, которые особенно хорошо приближаемы. Для этих направлений справедлив более сильный результат о возвращаемости.

Теорема 4.4. Рассмотрим обобщенный параллелограмм с углом $\alpha$. Пусть $\theta$ - такое направление, что $\|(\theta-p \alpha) / \pi\|<p^{-\mu}$ для бесконечно многих $p \in 2 \mathbb{N}$ $u\|(\theta+p \alpha) / \pi\|<p^{-\mu}$ для бесконечно многих $p \in 2 \mathbb{N}+1$. Тогда нижняя бох-размерность мнохсества $F_{\theta}$ не превосходит $1 /(\mu+1)$.

ДоказАТЕльство по сушеству такое же, как в теореме 4.1. Нужно только заменить неравенство (13) неравенством из условия теоремы и соответственно модифицировать (14).

Применим этот результат к перпендикулярньм траекториям в прямоугольных треугольниках. Здесь предполагается, что основание треугольника параллельно оси $x$.

СЛЕДСТВИЕ 4.5. Зафиксируем $\mu>1$ и рассмотрим прямоугольный треугольник с таким углом $\alpha / 2$, что приближение числа $\pi / 2$ орбитой угла $\alpha$ в смисле предыдущей теореми имеет порядок $\mu$. Тогда нижняя фрактальная размерность множества әлементов, перпендикулярных основанию треугольника и имеющих непериодические траектории, не превосходит $1 /(\mu+1)$.

Теорема 4.4 показывает, что для того чтобы получить лучший результат, чем в теореме 4.1, нужно рассматривать лучше приближаемые направления $(\mu>1)$. Введем множество

$$
\mathscr{C}_{s}:=\left\{\theta: \operatorname{dim}_{L B}\left(F_{\theta}\right) \leqslant s\right\}
$$


ТЕОРЕма 4.6. Зафиксируем произвольный обобщенный параллелограмм. Для любого $s \in[0,1 / 2]$

$$
\operatorname{dim}_{H} \mathscr{C}_{s} \geqslant \frac{s}{1-s}
$$

кроме того, $\mathscr{C}_{s}$ - остаточное мнохсество ьох-размерности 1.

ДокАЗАТЕльство. Первое утверждение непосредственно вытекает из предыдушей теоремы и теоремы 3.2 при $m=2$. Второе утверждение является прямьм следствием предложения 3.1 .

Теперь мы зафиксируем направление и будем варьировать обобщенньй параллелограмм. С этой целью предположим, что одна из сторон многоугольника зафиксирована, и будем отсчитывать направление $\theta$ от этого фиксированного направления. Заметим, что нашши оценки для $F_{\theta}$ будут зависеть только от угла $\alpha$ и больше ни от каких параметров обобщенного параллелограмма. Зафиксируем направление $\theta_{0}$. Рассмотрим множества

$$
\begin{aligned}
\mathscr{D}_{s}:=\left\{\alpha \in \mathbb{S}^{1}: \operatorname{dim}_{L B}\left(F_{\theta_{0}}\right)<s\right. \text { для всех } \\
\text { обобшенных параллелограммов с углом } \alpha\}, \\
\mathscr{E}_{s}:=\left\{\alpha \in \mathbb{S}^{1}: \operatorname{dim}_{L B}\left(F_{\text {перп }}\right)<s\right. \text { для } \\
\text { прямоугольного треугольника с углом } \alpha / 2\},
\end{aligned}
$$

где перп - направление, перпендикулярное одному из катетов.

Tеорема 4.7. (a) Ecлu $s \in[0,1 / 2]$, mo $\operatorname{dim}_{H} \mathscr{E}_{s} \geqslant 2 s$. При этом $\mathscr{E}_{s}-$ остаточное мнохество бох-размерности 1 .

(б) При заданном $\theta_{0}$ и при $s \in[0,1 / 2]$ выполнено неравенство $\operatorname{dim}_{H} \mathscr{D}_{s} \geqslant 2 s$. При әтом $\mathscr{D}_{s}$ - остаточное мнохсество ьох-размерности 1.

ДокАЗАтЕльство. Результаты о хаусдорфовой размерности непосредственно вытекают из теоремы 4.4 и теоремы 3.5 при $m=2$. Результаты о массивности и ящичной размерности являются прямым следствием предложения 3.3 .

Авторы благодарны Мартину Шмоллю и Анатолию Степину за полезные обсуждения.

\section{Список литературы}

1. Ruijgrok Th. Periodic orbits in triangular billiards // Acta Phys. Polon. B. 1991. V. 22. P. 955-981.

2. Boshernitzan M. Billiards and rational periodic directions in polygons // Amer. Math. Monthly. 1992. V. 99. P. 522-529.

3. Воробеи Я.Б., Гальперин Г.А., Степин А.М. Периодические бильярдные траектории в многоугольниках: механизмы рождения // УМН. 1992. Т. 47. №3. С. 9-74.

4. Cipra B., Hanson R., Kolan A. Periodic trajectories in right triangle billiards // Phys. Rev. E (3). 1995. V. 52. P. 2066-2071.

5. Gutkin E., Troubetzkoy S. Directional flows and strong recurrence for polygonal billiards // Proc. of the International congress of dynamical systems, Montevideo, Uruguay, 1995 / ed. F. Ledrappier et al. Harlow: Longman, 1996. P. 21-45.

6. Cassels J. W.S. An introduction to Diophantine approximation. Cambridge: Cambridge Univ. Press, 1957. 
7. Jarnik $V$. Diophantische Approximationen und Hausdorff Maß // Math. Sbornik. 1929. V. 36. P. 371-382.

8. Levesley J. A general inhomogeneous Jarnik-Besicovitch theorem // J. Number Theory. 1998. V. 71. P. $65-80$.

9. Troubetzkoy S. Recurrence and periodic billiard orbits in polygons // Preprint; http:// iml.univ-mrs/fr:80/ troubetz/pubs.html.

10. Falconer $K$. The geometry of fractal sets. Cambridge: Cambridge Univ. Press, 1985.

11. Pesin Ya. Dimension theory in dynamical systems. Chicago: Univ. of Chicago Press 1997.

12. Gutkin E. Billiard in polygons // Phys. D. 1986. V. 19. P. 311-333.

13. Gutkin E. Billiard in polygons: survey of recent results // J. Statist. Phys. 1996. V. 83. P. 7-26.

14. Tabachnikov S. Billiards // Panoramas et syntheses. Paris: Soc. Math. France, 1995.

15. Dodson M., Rynne B., Vickers J. Diophantine approximation and a lower bound for Hausdorff dimension // Mathematika. 1990. V. 37. P. 59-73.

16. Kra B., Schmeling J. Diophantine numbers, dimension and Denjoy maps // Acta Arith. (to appear); http://www.math.psu/edu/kra/publications.html.

Centre de Physique Théorique and

Institut de Mathématiques de Luminy, France;

Freie Universität Berlin and

Institut de Mathématiques de Luminy, France

E-mail: troubetz@iml.univ-mrs.fr, joerg@maths.lth.se
Поступила в редакцию

17.05.2001 и 21.06.2002 